\title{
On the Inverse Problem in Statistical Mechanics
}

\author{
N.M. Hugenholtz \\ Institute for Theoretical Physics, University of Groningen, Groningen, The Netherlands
}

\begin{abstract}
For quantum spin systems it is known that for a suitable space of potentials the equilibrium states are $W^{*}$-dense in the set of all translation invariant states. The problem discussed in this paper is how to recognize such equilibrium states and how to find the corresponding potential. A necessary and sufficient condition for a state to be an equilibrium state for some potential is given in Sect. 3 .
\end{abstract}

\section{Introduction}

The problem of equilibrium statistical mechanics is to determine the equilibrium state of a system for given temperature and given interaction (and possibly other parameters such as chemical potential, external field, etc.). Except for the most trivial models this is a formidable problem. Only in exceptional cases some correlation function can be calculated rigorously. In most cases one has to rely on approximation methods, such as perturbation theory. However, in the neighborhood of critical potentials even such approximation methods fail, or, if they give satisfactory results, it is not quite understood why they work.

Notwithstanding these computational difficulties the problem is well-defined, at least in the case of quantum lattice systems. The interaction between spins is determined by a potential. For each potential in a certain Banach space of potentials there is at least one equilibrium state. In case there is more than one equilibrium state for a given potential, this set is shown to be a simplex, so that each such state can be decomposed uniquely in terms of the set of extreme equilibrium states (the pure phases). The set of all equilibrium states for all possible potentials is $W^{*}$-dense in the set of all translation invariant states [1].

The inverse problem in statistical mechanics is to determine the potential for which a given state is in equilibrium. This problem has two aspects, an existence aspect and a computational aspect. As not every state is an equilibrium state for some potential, it is of interest to have sufficient conditions for a state to be an equilibrium state for some potential. Another related problem is how to determine this potential even if one knows that the state is in equilibrium. In other words, can 
one determine the potential with arbitrary precision by measuring correlation functions? These are not just academic questions since they also have practical interest. In the renormalization theory of critical phenomena, e.g., the so-called renormalization transformation is a transformation on potential space. In order to derive this transformation one in faced with the problem of finding the potential corresponding to a given state.

In this paper a modest attempt is made to discuss some aspects of these problems. We have limited ourselves to quantum lattice systems. These systems have the advantage over continuous systems that the problems can be well formulated in the framework of $C^{*}$-algebras. In Sect. 2 we prepare the ground with a rather extensive but necessary discussion of potentials. We stress in particular the use of unique potentials as introduced by Griffith and Ruelle [2]. A simple computation scheme to calculate the unique potential from an arbitrary potential can be found in the appendix. Section 3 gives a necessary and sufficient condition for a state to be an equilibrium state. In Sect. 4 we discuss the possibility of finding the potential for which a given state is in equilibrium.

\section{Spaces of Potentials}

We consider in this paper quantum spin one half systems. With each point $x$ of a $v$-dimensional cubic lattice $\mathbb{Z}^{v}$ we associate a 2-dimensional Hilbert space $\mathfrak{G}_{x}$. If $\Lambda \subset \mathbb{Z}^{v}$ is a finite subset of $\mathbb{Z}^{v}$, consisting of $|\Lambda|$ points, we define the tensor product

$$
\mathfrak{H}(\Lambda)=\prod_{x \in \Lambda} \otimes \mathfrak{S}_{x} .
$$

The algebra $\mathfrak{A}(\Lambda)$ of $2^{|\Lambda|} \times 2^{|\Lambda|}$ matrices acting on $\mathfrak{H}(\Lambda)$ contains the observables corresponding to $\Lambda$ as self-adjoint elements.

If $\Lambda_{1} \subset \Lambda_{2}$ we write $\mathfrak{H}\left(\Lambda_{2}\right)=\mathfrak{H}\left(\Lambda_{1}\right) \otimes \mathfrak{H}\left(\Lambda_{2} / \Lambda_{1}\right)$, and we shall identify $\mathfrak{A}\left(\Lambda_{1}\right)$ with $\mathfrak{A}\left(\Lambda_{1}\right) \otimes \mathbb{1}_{\Lambda_{2} / \Lambda_{1}}$. We then have $\mathfrak{A}\left(\Lambda_{1}\right) \subset \mathfrak{A}\left(\Lambda_{2}\right)$. Similarly, if $\Lambda_{1} \cap \Lambda_{2}=\emptyset$ the algebras $\mathfrak{A}\left(\Lambda_{1}\right)$ and $\mathfrak{U}\left(\Lambda_{2}\right)$ commute. The union $\mathfrak{A}_{L}$ of all $\mathfrak{A}(\Lambda)$ is the algebra of local observables. Its completion in the norm $\mathfrak{A}$ is the $C^{*}$-algebra of quasi-local observables. Translations, defined in an obvious manner, correspond to a representation of the additive group $\mathbb{Z}^{v}$ as automorphisms of $\mathfrak{A}$. For $a \in \mathbb{Z}^{v} \tau_{a}(A)$ is the observable obtained from $A$ by shifting over $a$. Clearly $\tau_{a}(\mathfrak{A}(\Lambda))=\mathfrak{A}(\Lambda+a)$.

In discussing a finite system the dynamics and thermal equilibrium properties are expressed by means of the Hamiltonian. In the case of an infinite system its role is taken over by the potential.

Definition 2.1. A translation-invariant potential $\Phi$ is a function $X \rightarrow \Phi(X)$ from the finite subsets of $\mathbb{Z}^{v}$ to the self-adjoint elements of $\mathfrak{A}$, such that
i. $\Phi(X) \in \mathfrak{A}(X)$,
ii. $\tau_{a}(\Phi(X))=\Phi(X+a)$.

We shall be interested in only such potentials where $\|\Phi(X)\|$ decreases sufficiently rapidly with increasing $X$. We define a norm

$$
\|\Phi\|_{1}=\sum_{0 \in X} \frac{\|\Phi(X)\|}{|X|}
$$

and consider the Banach-space $\mathfrak{B}_{1}=\left\{\Phi:\|\Phi\|_{1}<\infty\right\}$. 
This space is still too large for most applications and contains potentials with $\Phi(X)$ not sufficiently decreasing with increasing $|X|$ and $d(X)$ (the diameter of $X$ ). Smaller spaces can be defined as follows. Let $g$ be a function $X \rightarrow g(X)$ from the finite subsets of $\mathbb{Z}^{v}$ to the positive numbers, such that $g(X) \geqq 1, g(X+a)=g(X)$ and $g$ increases with increasing $|X|$ and/or $d(X)$. Then

and

$$
\|\Phi\|_{g}=\sum_{0 \in X} \frac{\|\Phi(X)\|}{|X|} g(X),
$$

Clearly $\mathfrak{B}_{g} \subset \mathfrak{B}_{1}$.

$$
\mathfrak{B}_{g}=\left\{\Phi:\|\Phi\|_{g}<\infty\right\}
$$

Since each term in (2.1) and (2.2) is invariant for translation of $X$ it is convenient to introduce the notation $\tilde{X}$ for the set $\left\{X+x, x \in \mathbb{Z}^{v}\right\}$. Then

and

$$
\|\Phi\|_{1}=\sum_{\tilde{X}}\|\Phi(\tilde{X})\|
$$

$$
\|\Phi\|_{g}=\sum_{\tilde{X}}\|\Phi(\tilde{X})\| g(\tilde{X})
$$

Potentials may be used to define the dynamics of the system. We shall call

$$
H(\Phi, \Lambda)=\sum_{X \subset \Lambda} \Phi(X)
$$

the Hamiltonian of the finite sublattice $\Lambda$. The time evolution is obtained by the limit

$$
\lim _{\Lambda \rightarrow \infty} e^{i H(\Phi, \Lambda) t} A e^{-i H(\Phi, \Lambda) t}=\alpha_{t}^{\Phi}(A) .
$$

As shown by Robinson and Ruelle $[3,4]$ this limit exists and gives rise to a strongly continuous one-parameter group of automorphisms of $\mathfrak{A}$ provided $\Phi \in \mathfrak{B}_{g}$, with $g$ satisfying the condition:

For $|X|$ sufficiently large and $\alpha>0, \quad g(X) \geqq \mathrm{e}^{\alpha|X|}$.

Potentials also play a fundamental role for the calculation of the thermodynamical functions: the average free energy per lattice point, the average energy per lattice point and the average entropy per lattice point. We consider any Banach space of potentials $\mathfrak{B}_{g}$ with $g(X) \geqq 1$. Without proof we mention the following wellknown facts.

I. The average free energy per lattice point

$$
f(\Phi)=\lim _{\Lambda \rightarrow \infty}-|\Lambda|^{-1} \log \left(\operatorname{Tr} e^{-H(\Phi, \Lambda)}\right)
$$

exists for all $\Phi \in \mathfrak{B}_{g}$ and has the properties

1. $\left|f\left(\Phi_{1}\right)-f\left(\Phi_{2}\right)\right| \leqq\left\|\Phi_{1}-\Phi_{2}\right\|_{g}$,

2. $f$ is concave.

II. For $\varrho$ translation-invariant and $\Phi \in \mathfrak{B}_{g}$ the average energy per lattice point

where $A_{\Phi}=\sum_{0 \in X} \frac{\Phi(X)}{|X|}$.

$$
\lim _{\Lambda \rightarrow \infty}|\Lambda|^{-1} \varrho(H(\Phi, \Lambda))=\varrho\left(A_{\Phi}\right),
$$


III. For $\varrho$ translation-invariant the average entropy per lattice point

$$
s(\varrho)=-\lim _{\Lambda \rightarrow \infty}|\Lambda|^{-1} \operatorname{Tr}\left(\varrho_{\Lambda} \log \varrho_{\Lambda}\right)
$$

exists. Here $\varrho_{\Lambda} \in \mathfrak{U}(\Lambda)$ is the density matrix corresponding to the restriction of $\varrho$ to $\mathfrak{A}(\Lambda)$. The function $s$ has the following properties

1. $0 \leqq s(\varrho) \leqq \log 2$,

2. $s$ is affine.

The equilibrium states for a given potential $\Phi \in \mathfrak{B}_{g}$ are defined by means of the variational principle, expressed in the following theorem:

Theorem 2.1[1].

$$
f(\Phi)=\min _{\varrho \in I}\left(\varrho\left(A_{\Phi}\right)-s(\varrho)\right) ;
$$

here I is the set of translation-invariant states of $\mathfrak{A}$. The set $I^{\Phi}$ is the set of states for which this minimum is reached. This set is not empty. If g satisfies condition (2.5), all states in $I^{\Phi}$ statisfy the KMS condition.

Whereas, according to this theorem, to each potential $\Phi$ there corresponds at least one equilibrium state, the inverse is not true, i.e., not every state is an equilibrium state for some potential. However, the variational equation (2.6) has the following inverse

$$
s(\varrho)=\inf _{\Phi \in \mathfrak{B}_{g}}\left(\varrho\left(A_{\Phi}\right)-f(\Phi)\right) .
$$

It is an immediate consequence of (2.6) that every equilibrium state $\varrho_{\Phi}$ for potential $\Phi$ defines a unique tangent plane of $f$ at $\Phi$. Indeed, one has

$$
f(\Phi+\Psi) \leqq \varrho_{\Phi}\left(A_{\Phi+\Psi}\right)-s\left(\varrho_{\Phi}\right)=f(\Phi)+\varrho_{\Phi}\left(A_{\Psi}\right),
$$

so that $\Psi \rightarrow \varrho_{\Phi}\left(A_{\Psi}\right)$ is a tangent plane of $f$ at $\Phi$. It can be shown [1] that also the reverse is true: to each tangent plane of $f$ at $\Phi$ there corresponds an equilibrium state from $I^{\Phi}$. This one-to-one correspondence between the set of equilibrium states and the set of tangent planes of $f$ will be of use in the following sections.

We shall now have another look at Definition 2.1 of potentials. This definition is not unique in the sense that different potentials may give rise to the same dynamics and the same equilibrium state. A trivial example is the following. If $\Phi \in \mathfrak{B}_{g}$ we define $\Phi^{\prime}=\Phi+\delta \Phi$, where $\delta \Phi(X)=C(X) \in \mathfrak{R}$, with

$$
\sum_{\tilde{X}}|C(\tilde{X})| g(\tilde{X})<\infty .
$$

Clearly $\alpha_{t}^{\Phi}=\alpha_{t}^{\Phi^{\prime}}$. Furthermore

$$
f\left(\Phi^{\prime}\right)=f(\Phi)+\sum_{\tilde{X}} C(\tilde{X}) .
$$

We see that the graph of $f$ contains a straight line, i.e., $f$ is not strictly concave:

$$
f(\Phi+\mu \delta \Phi)=f(\Phi)+\mu f(\delta \Phi) .
$$

Definition 2.2. Two potentials are called physically equivalent if they give rise to the same average energy and free energy, the same equilibrium states and the same dynamics. 
Proposition 2.2. Two potentials $\Phi_{1}$ and $\Phi_{2} \in \mathfrak{B}_{g}$ with $g$ satisfying (2.5) are physically equivalent if and only if $\varrho\left(A_{\Phi_{1}}\right)=\varrho\left(A_{\Phi_{1}}\right)$ for all $\varrho \in I$.

Proof. We have $f\left(\Phi_{1}\right)=\min _{\varrho \in I}\left(\varrho\left(A_{\Phi_{1}}\right)-s(\varrho)\right)=\min _{\varrho \in I}\left(\varrho\left(A_{\Phi_{2}}\right)-s(\varrho)\right)=f\left(\Phi_{2}\right)$. Consequently $I^{\Phi_{1}}=I^{\Phi_{2}}$; every state $\varrho \in I^{\Phi_{1}}=I^{\Phi_{2}}$ satisfies the K.M.S. condition with respect to $\alpha_{t}^{\Phi_{1}}$ and $\alpha_{t}^{\Phi_{2}}$. Since a state can only satisfy the K.M.S. condition with respect to one group of automorphisms, we conclude that $\alpha_{t}^{\Phi_{1}}=\alpha_{t}^{\Phi_{2}}$.

Instead of working with equivalence classes of physically equivalent potentials we prefer to make $\Phi$ unique by imposing some extra conditions. We follow the papers by Griffiths and Ruelle [2] and by Roos [5].

Definition 2.3. A unique translation-invariant potential $\Phi$ is a function $X \rightarrow \Phi(X)$ from the finite subsets of $\mathbb{Z}^{v}$ to the self-adjoint elements of $\mathfrak{A}$, such that

i. $\Phi(X) \in \mathfrak{U}(X)$;

ii. $\tau_{a}(\Phi(X))=\Phi(X+a)$;

iii. $\operatorname{Tr}^{(Y)} \Phi(X)=0, Y \subset X$,

where $\operatorname{Tr}^{(Y)}$ is the partial trace with respect to the Hilbert space $\mathfrak{H}(Y)$. It is shown in the appendix how an equivalent unique potential can be obtained from a given potential that does not satisfy iii. We shall assume in the sequel that potentials are as defined in Definition 2.3.

We shall now exhibit an interesting parallel between finite and infinite systems. In a finite system the set of Hamiltonians equals the set of self-adjoint elements of $\mathfrak{U}$, where $\mathfrak{U}$ is the set of all bounded operators on some Hilbert space $\mathfrak{H}$. We shall now show that there is a one-to-one correspondence between potentials and (equivalence classes of) self-adjoint elements of the algebra $\mathfrak{A}$.

Let $\tau$ be the unique tracial state of $\mathfrak{A}$ and $\mathfrak{F}$ the Hilbert space obtained as completion of $\mathfrak{U}$ with the scalar product $(A, B)=\tau\left(A^{*} B\right)$. The total set of linear subspaces $\left\{\mathfrak{A}(X), X \subset \mathbb{Z}^{v},|X|<\infty\right\}$ are not mutually orthogonal, but by means of an orthogonalization process we can construct mutually orthogonal subspaces $\{\mathfrak{F}(X)\}$, where for each $X \mathfrak{F}(X)=\{A \in \mathfrak{U}(X): A$ orthogonal to all $\mathfrak{U}(Y)$ for $Y \subsetneq X$. It is then easy to prove that, for $|X| \neq 0$,

$$
\mathfrak{F}(X)=\left\{A \in \mathfrak{U}(X): \operatorname{Tr}^{(Y)} A=0, \quad Y \subset X\right\} .
$$

Since $\mathfrak{F}=\sum_{X} \oplus \mathfrak{F}(X)$, there is for each $A \in \mathfrak{A}$ the unique decomposition $A=\sum_{X} A(X)$, where

i. $A(X) \in \mathfrak{U}(X)$,

ii. $\operatorname{Tr}^{(Y)} A(X)=0$ for all $Y \subset X,|X| \neq 0$.

If $A$ is non-local, the sum $\sum_{X} A(X)$ converges not only in the norm-topology of $\mathfrak{F}$ but also in the norm of $\mathfrak{A}$. This is seen as follows. For $\varepsilon>0$ there is a finite sublattice $\Lambda$ and $A^{\prime} \in \mathfrak{U}(\Lambda)$ such that $\left\|A^{\prime}-A\right\|<\frac{1}{2} \varepsilon$. But then $\left\|\tau^{\left(\Lambda^{c}\right)}\left(A^{\prime}-A\right)\right\|<\frac{1}{2} \varepsilon$, where $\tau^{\left(\Lambda^{c}\right)}$ is the partial trace with respect to $\mathfrak{A}\left(\Lambda^{c}\right)$. Hence $\left\|A^{\prime}-\sum_{X \subset A} A(X)\right\|<\frac{1}{2} \varepsilon$, so that $\left\|A-\sum_{X \subset \Lambda} A(X)\right\|<\varepsilon$. 
The decomposition $A=\sum_{X} A(X)$ enables one to define another, larger, norm:

$$
\|A\|_{1}=\sum_{X}\|A(X)\|
$$

with $\|A\|_{1} \geqq\|A\|$. Hence the Banach space $\mathfrak{U}_{1}=\left\{A \in \mathfrak{U}:\|A\|_{1}<\infty\right\}$ is a subset of $\mathfrak{A}$.

As long as we are interested in translation invariant states only we consider equivalence classes of observables that have the same expectation value for all states $\varrho \in I$. We define the closed linear subspace $\mathfrak{M} \subset \mathfrak{A}$ as the set $\{B \in \mathfrak{U}: \varrho(B)=0$ for all $\varrho \in I\}$. One proves without difficulty that $\mathfrak{M}$ is spanned by elements of the form $\tau_{x}(A)-A$ and also that $\mathfrak{M}=\left\{B \in \mathfrak{U}: \lim _{\Lambda \rightarrow \infty}|\Lambda|^{-1} \sum_{x \in \Lambda} \tau_{x}(B)=0\right\}$. Let $\mathfrak{X}=\mathfrak{U} / \mathfrak{M}$ be the set of equivalence classes. For $x \in \mathfrak{X}$, the norm is defined by

$$
\|x\|=\inf _{A \in x}\|A\| \text {. }
$$

With this norm $\mathfrak{X}$ is a Banach space.

Theorem 2.3: For $x \in \mathfrak{X}$, and $A \in X$,

$$
\|x\|=\lim _{\Lambda \rightarrow \infty}|A|^{-1}\left\|\sum_{x \in \Lambda} \tau_{x}(A)\right\| .
$$

For the proof, see reference [6].

It is immediately seen that there is a one-to-one correspondence between translation invariant elements of $\mathfrak{U}^{*}$ (the dual of $\mathfrak{A}$ ) and elements of $\mathfrak{X}^{*}$. If $Q$ is the canonical map of $\mathfrak{A}$ onto $\mathfrak{X}$, the correspondence between $\omega$ translation invariant $\in \mathfrak{A}^{*}$ and $\tilde{\omega} \in \mathfrak{X}^{*}$ is given by $\omega(A)=\tilde{\omega}(Q A)$.

We shall now show that there exists an isometric one-to-one mapping of $\mathfrak{B}_{1}$ into $\mathfrak{X}_{1}=\mathfrak{U}_{1} / \mathfrak{M}$, where $\mathfrak{U}_{1}$ is the Banach space defined above. If $A \in \mathfrak{A}_{1}$ we can define another element $\bar{A} \in \mathfrak{U}_{1}$ as follows.

$$
\bar{A}=A(\emptyset)+\sum_{x \ni 0} \frac{\bar{A}(X)}{|X|},
$$

where, for $X \neq \emptyset, \bar{A}(X)=\sum_{x} \tau_{-x}(A(X+x))$. One verifies immediately that $\bar{A}=A$ $(\bmod \mathfrak{M})$ and

$$
\begin{aligned}
& \text { i. } \bar{A}(X) \in \mathfrak{U}(X) ; \\
& \text { ii. } \tau_{a}(\bar{A}(X))=\bar{A}(X+a) ; \\
& \text { iii. } \operatorname{Tr}^{(Y)} \bar{A}(X)=0, \quad Y \subset X .
\end{aligned}
$$

Furthermore,

and

$$
\sum_{0 \in X} \frac{\|\bar{A}(X)\|}{|X|}<\infty
$$

$$
\|\bar{A}\|_{1}=|A(\emptyset)|+\sum_{0 \in X} \frac{\|\bar{A}(X)\|}{|X|} .
$$


Theorem 2.4. For $x \in \mathfrak{X}_{1}$, and $A \in x$,

(see [6]).

$$
\|x\|_{1}=\|\bar{A}\|_{1}
$$

Comparing the properties (2.8) with those in Definition 2.3 of the unique potential, we are led to the following isometry between $\mathfrak{B}_{1}$ and the subspace of traceless self-adjoint elements of $\mathfrak{X}_{1}$. For $\Phi \in \mathfrak{B}_{1}, Q A_{\Phi} \in \mathfrak{X}_{1}$ and $\|\Phi\|_{1}=\left\|Q A_{\Phi}\right\|_{1}$. That $A_{\Phi}$ is self-adjoint and traceless is immediately seen. Conversely, if $x=x^{*} \in \mathfrak{X}_{1}$ and $\tilde{\tau}(x)=0$, we have for each $A \in x: \tau(A)=A(\emptyset)=0$. We define the potential $\Phi_{x} \in \mathfrak{B}_{1}$ as follows: $\Phi_{x}(X)=\bar{A}(X)$, for $A \in X$. Again we have $\left\|\Phi_{x}\right\|_{1}=\|x\|_{1}$.

We shall from now on identify $\mathfrak{B}_{1}$ with the traceless self-adjoint elements of $\mathfrak{X}_{1}$. We shall, therefore, write $\varrho(\Phi)$ instead of $\varrho\left(A_{\Phi}\right)$. Having made this identification we can extend $\mathfrak{B}_{1}$ to $\mathfrak{B}$, where $\mathfrak{B}$ is the set of traceless self-adjoint elements of $\mathfrak{X}$. As shown in [6] the variational principle as formulated in Theorem 2.1 can be extended to $\mathfrak{B}$.

Before embarking in a discussion of the inverse problem we must discuss the question what is a space of reasonable potentials. The space $\mathfrak{B}$ is too large for many reasons. Using an argument due to Israel [7] one proves that for any $n$ different extremal states of $I$ there is a potential in $\mathfrak{B}$ for which these states are in equilibrium. This implies in particular that a given state $\in \mathscr{E}(I)$ is equilibrium for many different potentials. Since equilibrium states correspond to tangent planes of the average free energy $f$, we see that $f$ is not strictly concave. As follows from Proposition 2.2 this will not occur in smaller spaces $\mathfrak{B}_{g}$, with $g$ satisfying (2.5). These latter spaces are also more reasonable in the sense that they lead to dynamics. Nevertheless, condition (2.5), is not strong enough to ensure that $\mathfrak{B}_{g}$ has good properties. In fact, (2.5) suppresses the norm of $\Phi(X)$ for large $|X|$, but $\Phi(X)$ may well decrease very slowly as a function of $d(X)$. As has been pointed out by Daniels and Van Enter $[8,9]$, this leads to bad behavior of such potential spaces as regards phase diagrams. This can only be remedied by requiring that $g$ does not only increase with increasing $|X|$ but also with increasing diameter $d(X)$. We, therefore, consider $\mathfrak{B}_{g}$ to be a Banach space of short range potentials if $g$ satisfies (2.5) and

$$
\lim _{\Delta \rightarrow \infty} \inf _{\tilde{X} \neq \Delta} g(\tilde{X})=\infty .
$$

Here $\tilde{X} \subset \Delta$ means that for some $x X+x \subset \Delta$. Banach spaces of finite range potentials are defined as follows. If $\Delta \subset \mathbb{Z}^{v}$ is finite

$$
\mathfrak{B}_{\Delta}=\{\Phi: \Phi(\tilde{X})=0 \text { for } \tilde{X} \not \Delta\} .
$$

These spaces are finite dimensional.

\section{Characterization of Equilibrium States}

As discussed in Sect. 2 we have for arbitrary $\varrho \in I$ that $s(\varrho)=\inf _{\Phi \in \mathfrak{B}_{q}}(\varrho(\Phi)-f(\Phi))$. The state $\varrho$ is an equilibrium state for some potential in $\mathfrak{B}_{g}$ if an only if there is a $\Phi \in \mathfrak{B}_{g}$ such that $s(\varrho)=\varrho(\Phi)-f(\Phi)$. In geometrical terms the problem is the following: Let $f$ be a concave function on some Banach space $\mathfrak{B}$ and $\varrho$ a plane such that $\varrho(\Phi)+p \geqq f(\Phi)$ for some $p \in \mathbb{R}$. Is there a point $\Phi \in \mathfrak{B}$ for which $\varrho(\Phi)-f(\Phi)$ 
Fig. 1

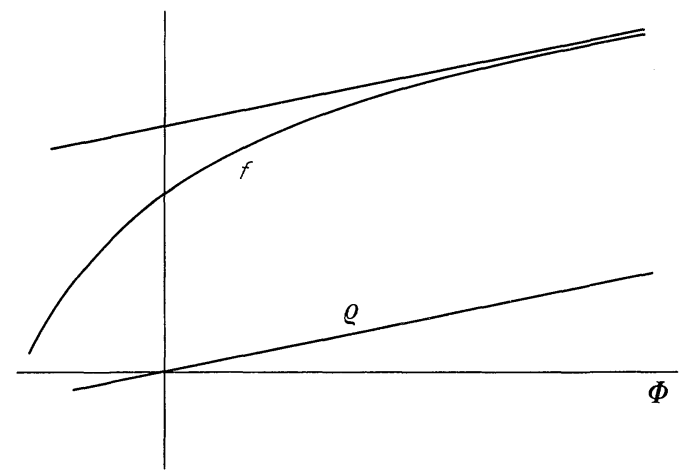

becomes minimal? This is not necessarily so, even if $\mathfrak{B}$ is finite dimensional, as is shown in Fig. 1 , where $\mathfrak{B}=\mathbb{R}$, and where $f$ has an asymptote parallel to $\varrho$. One could say loosely that there is a tangent point at infinity. A physical example of such a situation occurs when $\varrho$ is the ground state corresponding to some dynamical group $\alpha_{t}$. Then $T=0$, or $\beta=\infty$, which corresponds in our notation, where $\beta$ is absorbed in $\Phi$, with infinite $\Phi$. As we know the ground state is not an equilibrium state but is the $W^{*}$-limit of equilibrium states with decreasing temperatures.

The possibility of tangent points at infinity is excluded if we require of $\varrho$, that there is a positive number $M$ such that

$$
s(\varrho)=\inf _{\|\Phi\| \leqq M}(\varrho(\Phi)-f(\Phi)) .
$$

For finite dimensional $\mathfrak{B}$ it follows immediately that there is a minimum of $\varrho(\Phi)-f(\Phi)$, so that $\varrho$ is a tangent plane. To show this, we use the fact that the sphere $\{\Phi:\|\Phi\| \leqq M\}$ is compact, a property which is limited to finite dimensional spaces.

Nevertheless, the $\mathfrak{B}_{g}$, with $g$ satisfying (2.5) and (2.9) are sufficiently short range that the following theorem holds. As will be seen in the proof, condition (2.9) is essential.

Theorem 3.1. If $g$ satisfies the conditions (2.5) and (2.9), a necessary and sufficient condition for $\varrho$ to be an equilibrium state for some $\Phi \in \mathfrak{B}_{g}$ is that there is a positive number $M$ such that

$$
s(\varrho)=\inf _{\|\Phi\|_{g} \leqq M}(\varrho(\Phi)-f(\Phi)) .
$$

Proof. The necessity of the condition is obvious. We prove that the condition is sufficient. We first note that if $g$ satisfies the conditions (2.5) and (2.9), then so does $g_{n}$ for every $n \in \mathbb{N}$, where $g_{n}(\tilde{X})=g(\tilde{X})^{\frac{n}{n+1}}$. Secondly, the condition implies that there is a sequence $\left\{\Phi_{n}:\left\|\Phi_{n}\right\|_{g} \leqq M\right\}$ such that $s(\varrho)=\lim _{n \rightarrow \infty}\left(\varrho\left(\Phi_{n}\right)-f\left(\Phi_{n}\right)\right)$. Clearly, for every $\tilde{X}:|\tilde{X}|<\infty,\left\|\Phi_{n}(\tilde{X})\right\| \leqq M$. Since $\mathfrak{U}(X)$ is finite-dimensional, there is for each $X$ a converging subsequence. Using the diagonalization trick, there is a subsequence $\left\{n^{\prime}\right\}$ such that for each $\tilde{X}$ the sequence $\left\{\Phi_{n^{\prime}}(\tilde{X})\right\}$ converges. We show that the sequence $\left\{\Phi_{n^{\prime}}\right\}$ converges in $\mathfrak{B}_{g_{n}}$ for each $n$. 


$$
\begin{aligned}
\| \Phi_{n^{\prime}} & -\Phi_{m^{\prime}} \|_{g_{n}} \\
& =\sum_{\tilde{X}}\left\|\Phi_{n^{\prime}}(\tilde{X})-\Phi_{m^{\prime}}(\tilde{X})\right\| g_{n}(\tilde{X}) \\
& =\sum_{\tilde{X} \subset \Delta}\left\|\Phi_{n^{\prime}}(\tilde{X})-\Phi_{m^{\prime}}(\tilde{X})\right\| g_{n}(\tilde{X})+\sum_{\tilde{X} \notin \Delta}\left\|\Phi_{n^{\prime}}(\tilde{X})-\Phi_{m^{\prime}}(\tilde{X})\right\| g_{n}(\tilde{X}) \\
& \leqq \sum_{\tilde{X} \subset \Delta}\left\|\Phi_{n^{\prime}}(\tilde{X})-\Phi_{m^{\prime}}(\tilde{X})\right\| g_{n}(\tilde{X})+\left(\sup _{\tilde{X} \notin \Delta} g(\tilde{X})^{-\frac{1}{n+1}}\right) \sum_{\tilde{X} \phi \Delta}\left\|\Phi_{n^{\prime}}(\tilde{X})-\Phi_{m^{\prime}}(\tilde{X})\right\| g(\tilde{X}) \\
& \leqq \sum_{\tilde{X} \subset \Delta}\left\|\Phi_{n^{\prime}}(\tilde{X})-\Phi_{m^{\prime}}(\tilde{X})\right\| g_{n}(\tilde{X})+2 M \sup _{\tilde{X} \notin \Delta} g(\tilde{X})^{-\frac{1}{n+1}} .
\end{aligned}
$$

Using condition (2.9) we can choose $\Delta$ so large that the second term becomes $<\varepsilon / 2$. The first term, consisting of a finite sum, can be made smaller than $\frac{1}{2} \varepsilon$ by choosing $n^{\prime}$ and $m^{\prime}$ sufficiently large. We conclude that there is a $\Phi_{0} \in \mathfrak{B}_{g_{n}}$ such that $\lim _{n^{\prime} \rightarrow \infty}\left\|\Phi_{n^{\prime}}-\Phi_{0}\right\|_{g_{n}}=0$. Now $\varrho$ and $f$ are continuous on $\mathfrak{B}_{g_{n}}$, so that $s(\varrho)=\varrho\left(\Phi_{0}\right)-f\left(\Phi_{0}\right)$, which implies that $\varrho$ is equilibrium for $\Phi_{0}$. We shall now show that $\Phi_{0} \in \mathfrak{B}_{g}$. For each $n,\left\|\Phi_{0}\right\|_{g_{n}} \leqq M$. Since $\left\|\Phi_{0}\right\|_{g_{n}}$ increases with increasing $n$, the $\lim _{n}\left\|\Phi_{0}\right\|_{g_{n}}$ exists and is $\leqq M$. We have $\left\|\Phi_{0}\right\|_{g_{n}} \geqq \sum_{\tilde{X} \subset \Delta}\left\|\Phi_{0}(\tilde{X})\right\| g_{n}(\tilde{X})$, and hence $\lim _{n}\left\|\Phi_{0}\right\|_{g_{n}} \geqq \sum_{\tilde{X} \subset \Delta}\left\|\Phi_{0}(\tilde{X})\right\| g(\tilde{X})$, and by taking the limit $\Delta \rightarrow \infty$, we get that $\left\|\Phi_{0}\right\|_{g} \leqq M$.

Remark 1 . If $\varrho$ does not satisfy the condition of Theorem 3.1 we cannot conclude that $\varrho$ is a tangent plane at infinity (i.e. for a point $\Phi$ with one or more components infinite) like in the $T=0$ case. In fact, $\varrho$ may well be equilibrium for $\Phi$ in some larger space $\mathfrak{B}_{g^{\prime}}$, with $g^{\prime}<g$.

Remark 2. The criterion for equilibrium as given in this section is not practical in the sense that it can be tested. It assumes knowledge of the average free energy as a function of the potential. Therefore, it can be tested only if the main problem of equilibrium statistical mechanics is solved. A more useful criterion would involve conditions on the correlation functions.

\section{The Inverse Problem}

We assume in this section that the state $\varrho$ is equilibrium for some $\Phi \in B_{g}$, where $g$ satisfies conditions (2.5) and (2.9). Since $f$ is strictly concave on $B_{g}$, this potential is uniquely determined by $\varrho$. The question we shall discuss in this section is the following. Can we determine $\Phi$ approximately by performing a finite number of experiments? Mathematically speaking, doing measurements on $\varrho$ one determines a $W^{*}$-neighborhood of $\varrho$. As discussed extensively by Haag and Kastler [10], a $W^{*}$-neighborhood is very large, in the sense that every state $\varrho^{\prime}$ of $\mathfrak{U}$ contains in its representation a normal state in every weak neighborhood of $\varrho$. This fact can be understood as follows. A finite number of measurements gives information about $\varrho$ in some finite region $\Lambda$ : Having no further information one cannot distinguish between $\varrho$ and some totally different state $\varrho^{\prime}$ that coincides with $\varrho$ on $\Lambda$. 
The situation is, however, completely different if we restrict ourselves to translation-invariant states. In that case a finite number of measurements gives global information about $\varrho$.

Since, for finite $\Delta, B_{\Delta}$ is finite dimensional, the restriction of $\varrho$ to $B_{\Delta}$ can be determined by a finite number of measurements. Again, since $B_{\Delta}$ is finite dimensional, there is a unique $\Phi_{\Delta} \in B_{\Delta}$, and tangent plane $\varrho_{\Delta}$ at $\Phi_{\Delta}$ such that

$$
\varrho\left|B_{\Delta}=\varrho_{\Delta}\right| B_{\Delta} \text {. }
$$

This tangent point $\Phi_{\Delta}$ cannot be at infinity, as is seen without difficulty.

Let now $\left\{\Delta_{n}\right\}$ be an increasing sequence of finite sets, such that $\Delta_{n} \rightarrow \mathbb{Z}^{v}$, and let for each $n \Phi_{\Delta_{n}}$ be the potential determined by the restriction of $\varrho$ to $B_{\Delta_{n}}$. Intuitively we would expect the sequence to converge to $\Phi$. In the special case where $\Phi$ is of finite range this is indeed the case. If $\Phi \in B_{\Delta}$, clearly $\Phi_{\Delta_{n}}=\Phi$ for large enough $n$.

In general the series does not converge, as it need not be bounded. If the sequence $\left\|\Phi_{\Delta_{n}}\right\|_{g}$ is bounded we can conclude that $\Phi_{\Delta_{n}} \rightarrow \Phi$ as a consequence of the following proposition:

Proposition 4.1. If there is a sequence $\left\{\Phi_{n} \in B_{g}\right\}$ and $\Phi \in B_{g}$ such that

$$
W^{*} \lim _{n \rightarrow \infty} \varrho_{\Phi_{n}}=\varrho_{\Phi},
$$

where $\varrho_{\Phi_{n}}$ is an equilibrium state for potential $\Phi_{n}$, and if $\left\{\left\|\Phi_{n}\right\|_{g}\right\}$ is bounded, then

$$
\left\|\Phi_{n}-\Phi\right\|_{1} \rightarrow 0 \text {. }
$$

Proof. As is the proof of Theorem 3.1 there is a subsequence $\left\{\Phi_{n^{\prime}}\right\}$ and $\Phi^{\prime} \in B_{g}$, such that $\left\|\Phi_{n^{\prime}}-\Phi^{\prime}\right\|_{1} \rightarrow 0$. For each $n^{\prime}$,

$$
\varrho_{\Phi_{n^{\prime}}}\left(\Phi_{n^{\prime}}\right)-f\left(\Phi_{n^{\prime}}\right)-s\left(\varrho_{\Phi_{n^{\prime}}}\right)=0,
$$

and hence, taking the limit $n^{\prime} \rightarrow \infty$,

$$
\varrho_{\Phi}\left(\Phi^{\prime}\right)-f\left(\Phi^{\prime}\right)-s\left(\varrho_{\Phi}\right)=0,
$$

so that $\varrho_{\Phi}$ is equilibrium at $\Phi^{\prime}$. Since $f$ is strictly concave on $B_{g}$, we conclude that $\Phi=\Phi^{\prime}$ and $\left\|\Phi_{n}-\Phi\right\|_{1} \rightarrow 0$.

Remark. The approximation procedure as defined here is not a solution of the problem discussed in the introduction. The aim is to calculate the potential approximately from approximate knowledge of the correlation functions. The result of this section gives conditions under which this is possible, without giving a workable prescription.

\section{Appendix}

Let $\Phi$ be a potential according to Definition 2.1, with norm $\|\Phi\|_{g}=\sum_{0 \in X} \frac{\|\Phi(X)\|}{|X|} g(X)$ and $g$ such that $g(X) \geqq \exp (\alpha|X|)$. We assume in addition that for each $X \tau^{(X)} \Phi(X)=0$. For each $X$, we decompose $\Phi(X) \in \mathfrak{A}(X)$ according to the decomposition of elements of $\mathfrak{A}$ discussed in Sect. 2. We write

$$
\Phi(X)=\sum_{Y \subset X} \Phi(X, Y)
$$


where $\Phi(X, Y) \in \mathfrak{U}(Y)$ and $\tau^{\left(Y^{\prime}\right)} \Phi(X, Y)=0$ for $Y^{\prime} \subset Y$. We define

$$
\tilde{\Phi}=\left\{\tilde{\Phi}(Y)=\sum_{X \supset Y} \Phi(X, Y)\right\} .
$$

It is easy to verify that $\tilde{\Phi}$ is a potential according to Definition 2.3 . We shall compare the norms of $\tilde{\Phi}$ and $\Phi$. We define, for $X^{\prime} \subset X$,

$$
T\left(X, X^{\prime}\right)=\tau^{\left(X / X^{\prime}\right)}(\Phi(X)),
$$

so that $T(X, \emptyset)=0$ and $T(X, X)=\Phi(X)$. Furthermore $\left\|T\left(X, X^{\prime}\right)\right\| \leqq\|\Phi(X)\|$. Taking the partial trace of $\Phi(X)=\sum_{Y \subset X} \Phi(X, Y)$ with respect to $\mathfrak{A}\left(X / X^{\prime}\right)$ we obtain,
for $X^{\prime} \subset X$

$$
T\left(X, X^{\prime}\right)=\sum_{Y \subset X^{\prime}} \Phi(X, Y) .
$$

This set of equations can be solved for $\Phi(X, Y)$

$$
\Phi(X, Y)=\sum_{Y^{\prime} \subset Y}(-1)^{|Y|+\left|Y^{\prime}\right|} T\left(X, Y^{\prime}\right) .
$$

Hence

and

$$
\|\Phi(X, Y)\| \leqq 2^{|\mathrm{Y}|}\|\Phi(X)\|
$$

We get finally

$$
\|\tilde{\Phi}(Y)\| \leqq 2^{|Y|} \sum_{X \supset Y}\|\Phi(X)\|
$$

$$
\begin{aligned}
\|\tilde{\Phi}\|_{g^{\prime}} & =\sum_{Y \ni 0}\|\tilde{\Phi}(Y)\| g^{\prime}(Y) \leqq \sum_{Y \ni 0} 2^{|\mathrm{Y}|} g^{\prime}(Y) \sum_{X \supset Y}\|\Phi(X)\| \\
& =\sum_{X \ni 0}\|\Phi(X)\| \sum_{0 \in Y \subset X} 2^{|Y|} g^{\prime}(Y),
\end{aligned}
$$

and thus, if $g(X)=\sum_{0 \in Y \subset X} 2^{|Y|} g^{\prime}(Y)$,

$$
\|\widetilde{\Phi}\|_{g^{\prime}} \leqq\|\Phi\|_{g} .
$$

We see that $\tilde{\Phi} \in B_{g^{\prime}}$ if $\Phi \in B_{g}$, and $g(X) \geqq \sum_{0 \in Y \subset X} 2^{|Y|} g^{\prime}(Y)$. If, for example, we choose
$g^{\prime}(Y)=\exp (\gamma|Y|)$ with $\gamma>0$, then

$$
\sum_{0 \in Y \subset X} 2^{|Y|} e^{\gamma|Y|} \leqq 2^{|X|} e^{\gamma|X|} \sum_{0 \in Y \subset X} 1=2^{2|X| \perp-1} e^{\gamma|X|} .
$$

Hence inequality (1) is satisfied with $g^{\prime}(X)=\exp (\gamma|X|)$ and $g(X)=\exp (\alpha|X|)$, with $\alpha \geqq \gamma+2 \log 2$. This is a better estimate than the one given in reference [5].

\section{References}

1. Lanford, O.E. III, Robinson, D. W.: Statistical mechanics of quantum spin systems. III. Commun. Math. Phys. 9, 327-338 (1968)

2. Griffiths, R.B., Ruelle, D.: Strict convexity (continuity) of the pressure in lattice systems. Commun. Math. Phys. 23, 169-175 (1971)

3. Robinson, D. W.: Statistical mechanics of quantum spin systems. II. Commun. Math. Phys. 7, 337348 (1968) 
4. Ruelle, D.: Statistical mechanics. New York: W.A. Benjamin 1969

5. Roos, H.: Strict convexity of the pressure: a note on a paper of R.B. Griffiths and D. Ruelle. Commun. Math. Phys. 36, 263-276 (1974)

6. Hugenholtz, N.M.: $C^{*}$-algebras and statistical mechanics. In: Proceedings of the symposium in pure mathematics of the A.M.S., held in Kingston, Ontario, 1980 (to appear)

7. Israel, R.B.: Existence of phase transitions for long-range interactions. Commun. Math. Phys. 43, 59-68 (1975)

8. Daniëls, H.A. M., van Enter, A.C.D.: Differentiability properties of the pressure in lattice systems. Commun. Math. Phys. 71, 65-76 (1980)

9. van Enter, A.C.D.: A note on the stability of phase diagrams in lattice systems. Commun. Math. Phys. 79, 25-32 (1981)

10. Haag, R., Kastler, D.: An algebraic approach to quantum field theory. J. Math. Phys. 5, 848-861 (1964)

Communicated by R. Jost

Received December 15, 1981 